\title{
Optimization of a Collagen-Targeted PET Probe for Molecular Imaging of Pulmonary Fibrosis
}

\author{
Pauline Désogère ${ }^{1,2}$, Luis F. Tapias ${ }^{3}$, Tyson A. Rietz ${ }^{1}$, Nicholas Rotile ${ }^{1}$, Francesco Blasi ${ }^{1}$, Helen Day ${ }^{1}$, Justin Elliott ${ }^{3}$, \\ Bryan C. Fuchs ${ }^{4}$, Michael Lanuti ${ }^{3}$, and Peter Caravan ${ }^{1,2}$ \\ ${ }^{I}$ The Athinoula A. Martinos Center for Biomedical Imaging, Department of Radiology, Massachusetts General Hospital and Harvard \\ Medical School, Charlestown, Massachusetts; ${ }^{2}$ The Institute for Innovation in Imaging, Department of Radiology, Massachusetts \\ General Hospital and Harvard Medical School, Boston, Massachusetts; ${ }^{3}$ Division of Thoracic Surgery, Massachusetts General \\ Hospital and Harvard Medical School, Boston, Massachusetts; and ${ }^{4}$ Division of Surgical Oncology, Massachusetts General Hospital \\ and Harvard Medical School, Boston, Massachusetts
}

\begin{abstract}
There is a large unmet need for a simple, accurate, noninvasive, quantitative, and high-resolution imaging modality to detect lung fibrosis at early stage and to monitor disease progression. Overexpression of collagen is a hallmark of organ fibrosis. Here, we describe the optimization of a collagen-targeted PET probe for staging pulmonary fibrosis. Methods: Six peptides were synthesized, conjugated to a copper chelator, and radiolabeled with ${ }^{64} \mathrm{Cu}$. The collagen affinity of each probe was measured in a plate-based assay. The pharmacokinetics and metabolic stability of the probes were studied in healthy rats. The capacity of these probes to detect and stage pulmonary fibrosis in vivo was assessed in a mouse model of bleomycin-induced fibrosis using PET imaging. Results: All probes exhibited affinities in the low micromolar range $(1.6 \mu \mathrm{M}<\mathrm{Kd}<14.6 \mu \mathrm{M})$ and had rapid blood clearance. The probes showed 2- to 8-fold-greater uptake in the lungs of bleomycin-treated mice than sham-treated mice, whereas the distribution in other organs was similar between bleomycin-treated and sham mice. The probe ${ }^{64} \mathrm{Cu}-\mathrm{CBP} 7$ showed the highest uptake in fibrotic lungs and the highest target-to-background ratios. The superiority of ${ }^{64} \mathrm{Cu}-\mathrm{CBP} 7$ was traced to a much higher metabolic stability compared with the other probes. The specificity of ${ }^{64} \mathrm{Cu}-\mathrm{CBP} 7$ for collagen was confirmed by comparison with a nonbinding isomer. Conclusion: ${ }^{64} \mathrm{Cu}-\mathrm{CBP} 7$ is a promising candidate for in vivo imaging of pulmonary fibrosis.
\end{abstract}

Key Words: fibrosis; lung; type I collagen; ${ }^{64} \mathrm{Cu}$; PET imaging

J Nucl Med 2017; 58:1991-1996

DOI: 10.2967/jnumed.117.193532

$\mathbf{P}$ ulmonary fibrosis is a disease of nonreversible scarring resulting in cough, hypoxemia, and in many cases death. In pulmonary fibrosis, normal, healthy lung is replaced by fibrotic tissue (1). Idiopathic pulmonary fibrosis has a dismal prognosis, with a median survival of only approximately $3 \mathrm{y}$ after diagnosis, and the prognosis for certain other fibrotic lung disease is only marginally better. There is significant heterogeneity with respect to disease

Received Mar. 22, 2017; revision accepted Jun. 1, 2017.

For correspondence or reprints contact: Peter Caravan, Athinoula A. Martinos Center for Biomedical Imaging, Department of Radiology, Massachusetts General Hospital and Harvard Medical School, Bldg. 149, Rm. 2301, 13th St., Charlestown, MA 02129.

E-mail: caravan@nmr.mgh.harvard.edu

Published online Jun. 13, 2017.

COPYRIGHT (C) 2017 by the Society of Nuclear Medicine and Molecular Imaging. progression within pulmonary fibrosis, however, because some patients exhibit only slow declines in lung function, whereas others experience very rapidly progressive disease. There is a critical need for new methods of identifying fibrosis, staging disease, monitoring change in disease, and characterizing disease activity.

Fibrosis, regardless of its cause or location, is characterized by excess deposition of collagens, primarily type I collagen, and other extracellular matrix proteins in the parenchyma (2). There are currently few molecular imaging approaches to assess fibrosis (3). We previously reported a 16-amino-acid disulfide-bridged cyclic peptide that was identified via phage display to recognize type I human collagen. This peptide was functionalized with 3 gadolinium chelates to provide MR signal enhancement, and the resulting probe EP-3533 showed excellent ability to detect and stage disease in preclinical models of cardiac $(4,5)$, hepatic $(6-8)$, and pulmonary fibrosis (9). On the basis of our positive MR findings with EP-3533, we sought to develop an analogous PET probe to quantify pulmonary fibrosis. PET is frequently used to characterize lung pathology. In the absence of lung architectural distortion, which may be absent early in fibrotic lung diseases, fibrosis may not be able to be distinguished from alternative x-ray-attenuating processes, such as inflammation, by high-resolution CT. By combining collagen-specific PET with high-resolution CT, however, it may be possible to determine whether specific opacities and patterns identified by high-resolution CT are produced by a fibrosing disease (10). In addition, the development of PET molecular imaging probes offers a shorter and more economic path to clinical translation compared with MR probes.

\section{MATERIALS AND METHODS}

\section{Synthesis, Radiochemistry, and Affinity of Collagen-Binding Probes (CBPs)}

We synthesized a series of peptides and the corresponding 1,4,7triazacyclononane-1-glutaric acid-4,7-acetate (NODAGA) conjugates and then reacted with ${ }^{64} \mathrm{CuCl}_{2}$. The synthetic route was analogous for each probe. All chemicals were purchased commercially and used without further purification. The 1,4,7-triazacyclononane was purchased from Chematech. The peptide precursors of CBP7 and CBP11 were custom-made by American Peptide. ${ }^{64} \mathrm{CuCl}_{2}$ was obtained from the University of Wisconsin. ( $\left.{ }^{\mathrm{Bu}}\right)_{3}$ NODAGA-NHS was synthesized inhouse following a published procedure (11). All intermediates and final compounds were purified by high-performance liquid chromatography using a Phenomenex Luna C18 column $(250 \mathrm{~mm} \times 21.2 \mathrm{~mm} \times 5 \mu \mathrm{m})$ and a gradient of water/acetonitrile with $0.1 \%$ trifluoroacetic acid. All 
intermediates and final compounds were characterized by liquid chromatography-mass spectrometry using a Phenomenex Kinetex C18 column $(150 \mathrm{~mm} \times 4.6 \mathrm{~mm} \times 5 \mu \mathrm{m})$ and a gradient of water/acetonitrile with $0.1 \%$ formic acid (Supplemental Fig. 1; Supplemental Tables 1-2 [supplemental materials are available at http://jnm.snmjournals.org]). The chemical purities of these compounds were greater than $97 \%$.

Peptides on resin were synthesized by standard Fmoc chemistry using solid-phase peptide synthesis on a CEM microwave peptide synthesizer on a $0.25 \mathrm{mmol}$ scale. The peptides Pep(n) were cleaved with a cocktail comprising trifluoroacetic acid/methanesulfonic acid (70\% in water)/triisopropylsilane/dodecanethiol, then precipitated in diethyl ether. Each Pep(n) was dissolved in a solution of dimethylsulfoxide/ water $(1 / 15,16 \mathrm{~mL})$, and the solution was adjusted to $\mathrm{pH} 5.5$ with a $0.1 \mathrm{M}$ solution of sodium hydroxide. The cyclization reactions were stirred at room temperature for $24 \mathrm{~h}$. The cyclized peptides cPep(n) were then coupled to tri-tert-butyl protected $\left({ }^{\mathrm{t}} \mathrm{Bu}\right)_{3}$ NODAGA-NHS in dimethylformamide, at $\mathrm{pH} 6.5$, in the presence of diisopropylethylamine to give $\left({ }^{\mathrm{t}} \mathrm{Bu}\right)_{3}$ NODAGA-cPep(n) after $24 \mathrm{~h}$. Deprotection of the tertbutyl groups with the cleavage cocktail described above resulted in the synthesis of NODAGA-cPep(n). NODAGA-cPep(n) was then isolated by precipitation in diethylether and purified. Reaction of NODAGAcPep(n) with an excess of ${ }^{n a t} \mathrm{CuSO}_{4}$ in $10 \mathrm{mM}$ sodium acetate buffer $(\mathrm{pH} 5.5)$ resulted in the synthesis of the nonradioactive surrogates CBPn $(n=1,3,5,6,7,11)$.

The general procedure for the radiolabeling of CBPn $(n=1,3,5$, 6) was as follows: ${ }^{64} \mathrm{CuCl}_{2}(3.7-14.8 \mathrm{MBq}$, in $30 \mu \mathrm{L})$ was diluted with $90 \mu \mathrm{L}$ of pH 5.1 ammonium acetate buffer $(40 \mathrm{mM})$. A $10-\mu \mathrm{L}$ aliquot of a $0.1 \mathrm{mM}$ ligand solution (in sodium acetate buffer, $\mathrm{pH} 4.1$ ) was added, and the reaction mixture was heated at $60^{\circ} \mathrm{C}$ for $15 \mathrm{~min}$. For CBP7 and CBP11, ${ }^{64} \mathrm{CuCl}_{2}$ was diluted with $90 \mu \mathrm{L}$ of $\mathrm{pH} 8.0$ sodium citrate buffer $(10 \mathrm{mM})$. A $10-\mu \mathrm{L}$ aliquot of a $0.1 \mathrm{mM}$ ligand solution (in 4-[2-hydroxyethyl]-1-piperazineethanesulfonic acid, $\mathrm{pH}$ 7.4) was added, and the reaction mixture was stirred at room temperature for $5 \mathrm{~min}$. After reaction, the probe was purified with a Sep-Pak C18 cartridge (Waters). The radiochemical purities of the final solutions were $99 \%$ or greater as determined by radio-high-performance liquid chromatography (HPLC) with a Phenomenex Luna C18 column $(150 \mathrm{~mm} \times 4.6 \mathrm{~mm} \times 5 \mu \mathrm{m})$ using a gradient of water/acetonitrile with $0.1 \%$ trifluoroacetic acid (Supplemental Fig. 2). The pH of the final solutions was adjusted to 7.4 using phosphate-buffered saline, and the radiolabeled CBPn were diluted in sucrose $(80 \mathrm{mM}$ in water) before injection into animals.

Collagen-binding isotherms were obtained by following a method previously reported (4).

\section{Pharmacokinetics and Metabolic Stability}

Pharmacokinetics and metabolic stability were obtained by following a method previously reported (12).

\section{Animal Model and Probe Administration}

All experiments were performed in accordance with National Institutes of Health Guidelines for the Care and Use of Laboratory Animals (13) and with the ARRIVE guidelines (14), and were approved by the Institution's Animal Care and Use Committee at Massachusetts General Hospital. A total of 60 mice and 6 rats were used in this study. Pulmonary fibrosis was induced in 10-wk-old male C57/ BL6 mice (20-28 g, Charles River Laboratories, $n=32$ ) by transtracheal administration of bleomycin $(2.5 \mathrm{U} / \mathrm{kg})$ in $50 \mu \mathrm{L}$ of phosphatebuffered saline under direct vision using a small cervical incision (15). Sham animals $(n=28)$ received the analogous volume of vehicle (phosphate-buffered saline). The mice were injected 13-14 d after bleomycin instillation by intrafemoral catheter with approximately 3.7 $\mathrm{MBq}$ in approximately $0.1 \mathrm{~mL}$ of the dose solution measured with a dose calibrator (CRC-25PET; Capintec).

\section{Small-Animal PET/CT Imaging and Analysis}

Animals were placed in a small-animal PET/CT scanner (Triumph; TriFoil Imaging), equipped with inhalation anesthesia and heating pad. Each animal was anesthetized with isoflurane (4\% for induction, $1 \%-1.5 \%$ for maintenance in medical air). After placement of an indwelling catheter in the femoral vein for probe administration, mice were positioned in the PET/CT scanner and the probe was given as a bolus as the PET acquisition began. Mice were imaged continuously by PET for $120 \mathrm{~min}$. A whole-body CT scan was obtained either immediately before or immediately after the PET acquisition, and the mice were then euthanized at $150 \mathrm{~min}$ after injection. Instruments were calibrated with phantoms containing small known amounts of radioactivity. Isotropic $(0.3 \mathrm{~mm}) \mathrm{CT}$ images were acquired over $6 \mathrm{~min}$ with 512 projections with 3 frames per projection (exposure time per frame, $\sim 200 \mathrm{~ms}$; peak tube voltage, $70 \mathrm{kV}$; tube current, $177 \mathrm{~mA}$ ). PET and CT images were reconstructed using the LabPET software (TriFoil Imaging), and the CT data were used to provide attenuation correction for the PET reconstructions. The PET data were reconstructed using a maximum-likelihood expectation maximization algorithm run over 30 iterations to a voxel size of $0.5 \times 0.5 \times 0.6 \mathrm{~mm}$. For the pharmacokinetic analyses, the PET data were reconstructed in 1-min (first 10 frames), 3-min (next 10 frames), and 10-min (last 8-10frames) intervals out to 120 min after injection. Reconstructed PET/ CT data were quantitatively evaluated using the AMIDE software package (16). For each PET scan, volumes of interest were drawn over major organs on decay-corrected whole-body coronal images. The radioactivity concentration within organs was obtained from mean pixel values within the volume of interest and converted to counts per milliliter per minute and then divided by the injected dose (ID) to obtain an imaging volume of interest-derived percentage injected radioactive dose per cubic centimeter of tissue $\left(\% \mathrm{ID} / \mathrm{cm}^{3}\right)$.

\section{Biodistribution Protocol}

The lungs, blood, urine, heart, liver, left rectus femoris muscle, spleen, small intestine, kidneys, and left femur bone were collected from all animals $150 \mathrm{~min}$ after probe injection. The tissues were weighed, and radioactivity in each tissue was measured on a $\gamma$-counter (Wizard2 Auto Gamma; PerkinElmer). Tracer distribution was presented as \% ID per gram (\%ID/g) for all organs. For the lungs, tracer was also presented as \% ID per lung (\%ID/lung).

\section{Histology and Quantification of Collagen}

For histologic purposes, 1 of the 2 lungs was inflated and fixed with $10 \%$ formalin, embedded in paraffin, cut into 5- $\mu \mathrm{m}$-thick sections, and stained with either hematoxylin and eosin or picrosirius red with a counterstain of fast green. The remaining lung was hydrolyzed in a concentrated hydrochloric acid solution, and the amount of hydroxyproline was quantified by HPLC after a 2-step derivatization process (17).

\section{Statistics}

Data were expressed as mean \pm SEM. Differences between groups were compared using the Student $t$ test and ANOVA, followed by the Tukey post hoc test. A $P$ value of less than 0.05 was considered significant.

\section{RESULTS}

\section{Synthesis, Radiochemistry, and Affinity of CBPs}

The general synthetic route for the probes is shown in Figure 1. CBP1, CBP3, CBP5, and CBP6 were obtained by reaction of the ligands with ${ }^{64} \mathrm{CuCl}_{2}$ in yields of $99 \%$ in ammonium acetate buffer (pH 5.1). For CBP7 and CBP11, considerable improvements in radiochemical yield were obtained when the labeling was performed 


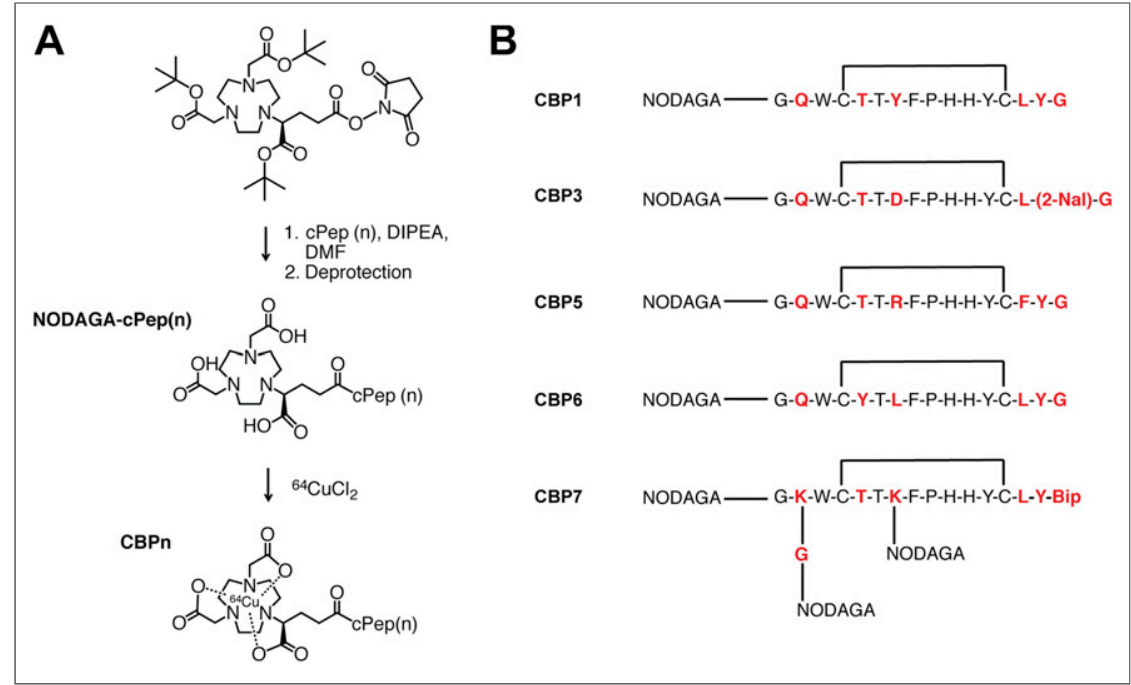

FIGURE 1. (A) General protocol for synthesis of CBPs. (B) Structures of CBP1, CBP3, CBP5, CBP6, and CBP7. Bip = 4,4'-biphenylalanine; 2-Nal = 2-napthylalanine.

at $\mathrm{pH} 8$ in sodium citrate $(10 \mathrm{mM})$. The reactions were quantitative, with a specific activity ranging from 6 to $18 \mathrm{GBq} / \mu \mathrm{mol}$.

CBP1, CBP3, CBP5, CBP6, and CBP7 exhibited moderate affinity and nonsaturable binding to type I collagen ranging from 1.6 to $14.6 \mu \mathrm{M}$ (Supplemental Table 3). The low micromolar affinities displayed by these 5 new copper compounds are similar to the affinity of the parent ligand EP-3533, which was previously reported to be $1.8 \mu \mathrm{M}(18)$. CBP11 is the D-Cys analog of CBP7 and has no affinity for collagen $(\mathrm{Kd}>100 \mu \mathrm{M})$.

\section{Pharmacokinetics and Metabolism in Rats}

The ${ }^{64} \mathrm{Cu}$ blood clearance profile was similar for all 5 probes (Supplemental Fig. 3), with elimination blood half-lives ranging from 18 to 23 min (Supplemental Table 4). Radio-HPLC analyses of rat serum after probe injection (15 and 60 min after injection) are shown in Figure 2A. CBP1, CBP3, CBP5, and CBP6 undergo decomposition into less hydrophobic metabolites due to peptidase activity. Metabolism was extremely rapid for CBP1, CBP3, and CBP5 with less than $50 \%$ of intact probe detected in the blood at $15 \mathrm{~min}$ after injection (Supplemental Fig. 4A). CBP6 was still largely intact at 15 min after probe injection (percentage of intact probe $>80$; Supplemental Fig. 4B) and then further metabolized in the next $45 \mathrm{~min}$. $\mathrm{CBP7}$ was completely intact in the serum at $60 \mathrm{~min}$ after injection and indeed highly stable over time: more than $80 \%$ of the circulating

$\left(0.95 \pm 0.14\right.$ vs. $0.24 \pm 0.1 \% \mathrm{ID} / \mathrm{cm}^{3}$, $P<0.0001$; Fig. 2B; Supplemental Fig. 5). One-way ANOVA between activity values in fibrotic tissues with the different probes revealed strong statistical differences. The signal in the lungs of fibrotic mice injected with $\mathrm{CBP7}$ was significantly higher than the signal in the lungs of the fibrotic animals injected with CBP3 $(P=0.0047)$, CBP5 $(P=0.0071)$, and CBP6 $(P=0.0009)$. The uptake in the fibrotic lungs ranged from 1.4- to 7-fold higher in the animals that received CBP7 than the animals that received the other probes $(P<0.05)$.

\section{Biodistribution in Mice with Pulmonary Fibrosis and in Sham Animals of 5 CBPs}

After PET/CT imaging, each probe was further assessed ex vivo by directly sampling tissues of interest (Supplemental Tables 5-9). Each probe showed greater lung uptake in bleomycin-treated mice (fibrotic) than sham mice, and this increased uptake in fibrotic lung ranged from 2- to 8-fold that of the sham (Fig. 2C; Table 1). The difference was statistically higher for CBP7 $(0.017 \pm 0.003$ vs. $0.107 \pm 0.022$, sham vs. bleomycin-treated, $P<0.0001)$. Among the 5 probes, CBP7 showed the highest uptake in fibrotic lungs $(P<0.05)$; there was no significant difference in the normal lung uptake between probes.

Apart from the lungs, all the probes showed a similar nontarget uptake in control and bleomycin-treated animals. Uptake in the liver for CBP3, CBP5, CBP6, and CBP7 $(2<\%$ ID/g $<4)$ may be attributed to a transmetallation phenomenon by endogenous proteins. All the probes were excreted pri-
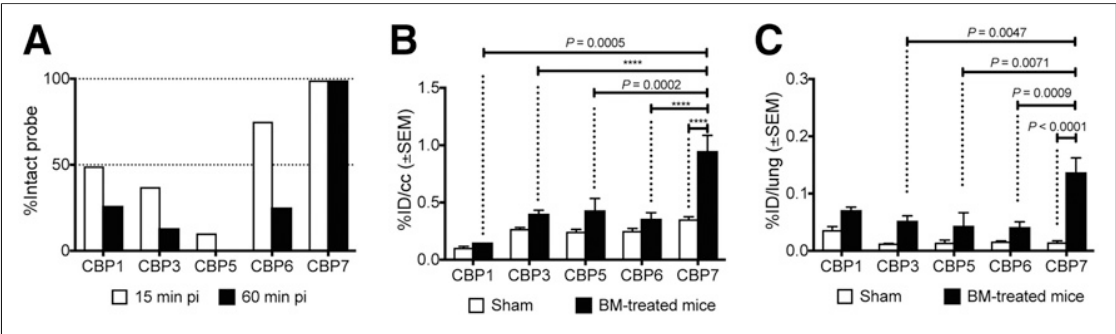

FIGURE 2. (A) Metabolic stability of each probe estimated from HPLC analysis of serum samples in rat at 15 and 60 min after probe injection. (B) Mean whole lung PET activity values in sham and bleomycin-treated mice (2 wk after bleomycin instillation) for CBP1, CBP3, CBP5, CBP6, and CBP7 (100-120 min after injection). (C) Ex vivo lung uptake in sham and bleomycin-treated mice (2 wk after bleomycin instillation) for CBP1, CBP3, СBP5, CBP6, and CBP7 at 150 min after probe injection. ${ }^{\star \star \star \star} P<0.0001$. BM $=$ bleomycin. marily through the kidneys. Similar to most peptide-based probes, we observed some retention in the kidneys. CBP7 exhibited the highest uptake in the kidneys, 2- to 8-fold higher than the other probes in both sham $(P<0.0001)$ and bleomycin-treated animals $(P<0.0001)$.

\section{Histology}

All of the bleomycin-treated mice had histopathologic findings consistent with substantial pulmonary fibrosis, including excessive deposition of collagen in the extracellular matrix as shown by picrosirius red staining (Fig. 3A), whereas the sham animals showed 
TABLE 1

Lung-to-Tissue Ratio in Bleomycin-Treated Mice at 150 Minutes After Injection

\begin{tabular}{lccccc}
\hline \multicolumn{1}{c}{ Organ } & CBP1 $(n=3)$ & CBP3 $(n=6)$ & CBP5 $(n=6)$ & CBP6 $(n=6)$ & CBP7 $(n=7)$ \\
\hline Blood & $3.98 \pm 0.96$ & $4.59 \pm 1.60$ & $3.88 \pm 2.26$ & $2.43 \pm 0.34$ & $2.41 \pm 0.85$ \\
Heart & $4.61 \pm 0.75$ & $5.00 \pm 0.77$ & $4.50 \pm 1.97$ & $3.46 \pm 0.22$ & $4.15 \pm 1.49$ \\
Liver & $0.48 \pm 0.13$ & $0.27 \pm 0.02$ & $0.12 \pm 0.07$ & $0.23 \pm 0.05$ & $0.55 \pm 0.07$ \\
Muscle & $5.23 \pm 3.75$ & $9.57 \pm 2.89$ & $14.26 \pm 6.49$ & $6.67 \pm 1.39$ & $11.91 \pm 5.50$ \\
Spleen & $2.68 \pm 0.56$ & $0.91 \pm 0.17$ & $0.38 \pm 0.24$ & $0.78 \pm 0.18$ & $1.02 \pm 0.22$ \\
Small intestine & $1.09 \pm 0.16$ & $1.08 \pm 0.37$ & $1.12 \pm 0.75$ & $0.64 \pm 0.10$ & $1.53 \pm 0.34$ \\
Kidney & $0.03 \pm 0.01$ & $0.05 \pm 0.01$ & $0.02 \pm 0.02$ & $0.04 \pm 0.01$ & $0.02 \pm 0.01$ \\
Bone & $3.56 \pm 2.56$ & $4.33 \pm 1.07$ & $2.00 \pm 1.48$ & $1.54 \pm 0.19$ & $2.62 \pm 0.53$ \\
& & & & & \\
\hline
\end{tabular}

no signs of pulmonary disease. These results demonstrated that the bleomycin model successfully induces pulmonary fibrosis in mice, accompanied by excessive deposition of collagen, including type I, in the extracellular matrix.

\section{Collagen Quantification}

Hydroxyproline is a quantitative biomarker of collagen (19). When all mice were compared, the hydroxyproline content was significantly higher in the lungs of the bleomycin-treated mice than in the lungs of the sham animals (Supplemental Fig. 6A). Ex vivo analysis showed a strong correlation between the injected dose per lung and the hydroxyproline content for CBP3 $\left(r^{2}=0.62, P<\right.$ 0.001, Fig. 3B), CBP5 $\left(r^{2}=0.78, P<0.01\right.$, Fig. 3C), CBP6 $\left(r^{2}=0.83, P<0.001\right.$, Fig. 3D), and CBP7 $\left(r^{2}=0.74, P<0.0001\right.$, Fig. 3E). For CBP1, the correlation between $\%$ ID/lung and

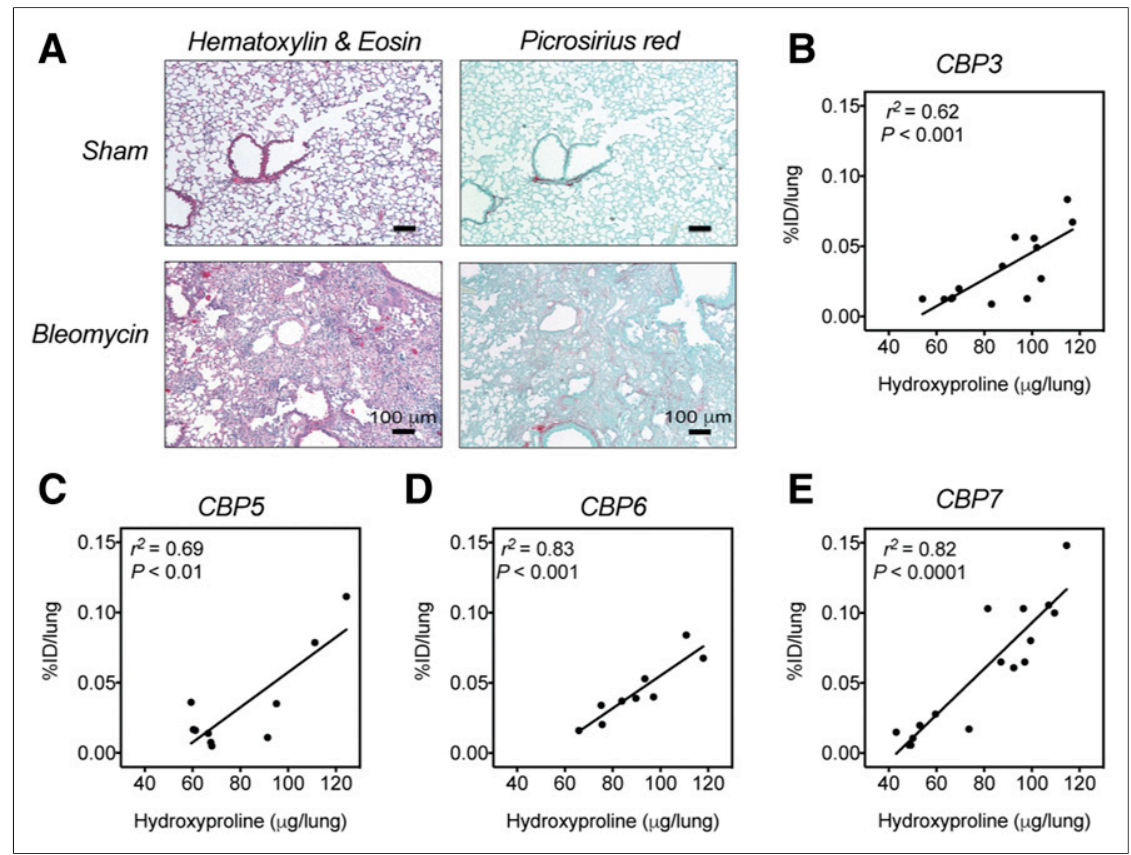

FIGURE 3. (A) Representative images of lung tissue stained with hematoxylin and eosin and picrosirius red $(\times 10$; scale bar, $100 \mu \mathrm{m})$ for sham mice and for bleomycin-treated mice $(2 \mathrm{wk}$ after bleomycin instillation). (B-E) Correlation between hydroxyproline content as measure of total lung collagen and \%ID/lung in sham and bleomycin-treated mice, 2 wk after instillation of bleomycin or vehicle and $150 \mathrm{~min}$ after probe injection. hydroxyproline content was much weaker $\left(r^{2}=0.11\right.$, Supplemental Fig. 6B).

\section{Specificity of CBP7}

To demonstrate the specificity of CBP7 binding to collagen, the isomer CBP11 was prepared. CBP11 differs from CBP7 only in the chirality of the cysteine residue at position X4 (D-Cys for CBP11). CBP11 was investigated in bleomycin-treated mice and controls. There was no difference in lung uptake between a bleomycintreated animal and a sham injected with CBP11 (Fig. 4A). This was confirmed by quantification of the PET data (Fig. 4B) and the ex vivo lung uptake (Fig. 4C). The biodistribution of CBP11 was assessed in sham animals and bleomycin-treated animals at $2.5 \mathrm{~h}$ after injection, and compared with CBP7 (Fig. 5; Supplemental Table 10). CBP11 uptake was similar in fibrotic lungs and in control lungs and showed a similar nontarget uptake in control and bleomycin-treated animals. CBP11 concentration in blood was similar to that of CBP7, as may be expected for the isomers in both cohorts. There was a trend toward slower blood clearance in the bleomycin-treated mice compared with sham mice for both CBP7 and CBP11, presumably because of disease; however, the increased uptake of CBP7 in the lungs of bleomycin-treated mice was much higher than could be accounted for by increased blood activity in the lungs. There was no statistically significant difference in CBP7 and CBP11 uptake in lung, kidney, spleen, heart, and liver in sham animals. In fibrotic lungs, CBP7 uptake was 3-fold higher than CBP11 uptake $(P=0.0047)$.

\section{DISCUSSION}

Herein, we present the synthesis and evaluation of 5 CBPs, CBP1, CBP3, CBP5, CBP6, and CBP7, for detecting pulmonary fibrosis in a murine model. We used a core peptide that has high affinity and specificity for collagen (4). We previously used the MR probe EP-3533 to demonstrate specific MR imaging of pulmonary collagen, but EP-3533 


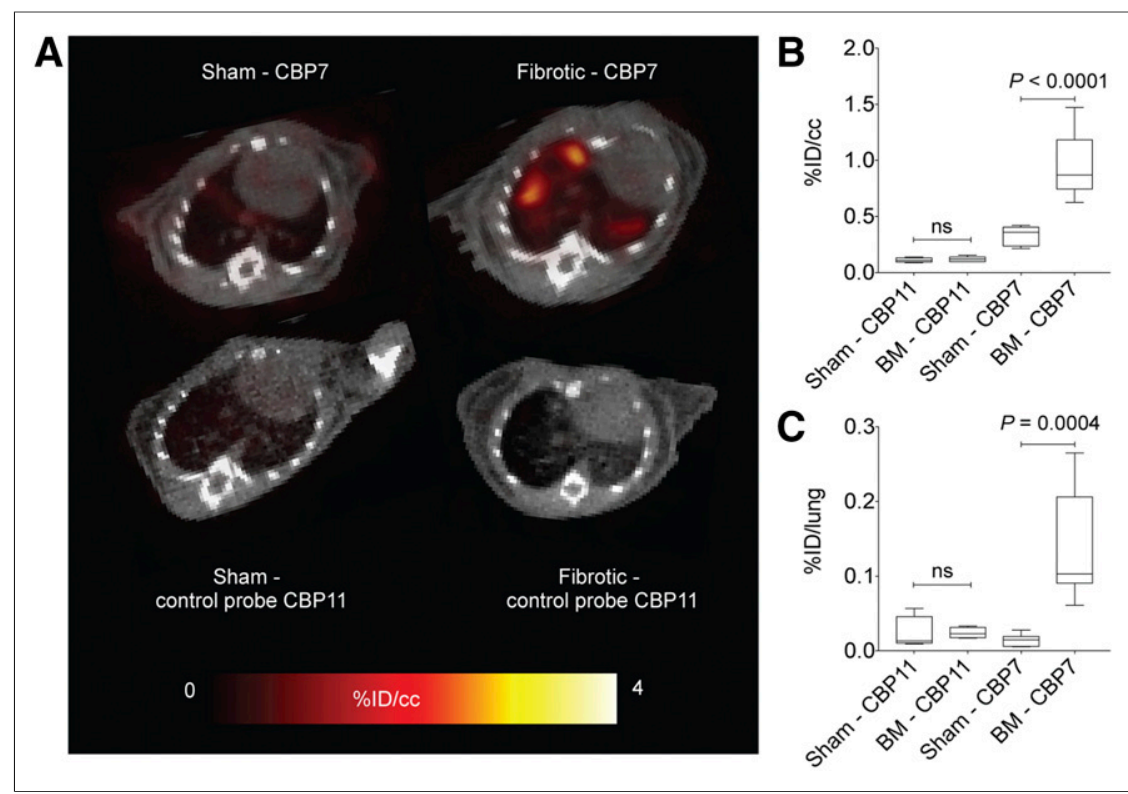

FIGURE 4. (A) Representative fused PET/CT images (axial view) of sham and bleomycin-treated animals injected with CBP7 (top) and CBP11 (bottom). Color scale image shows PET image from integrated data $100-120 \mathrm{~min}$ after probe injection. (B) Mean lung PET activity values $\left(\% \mathrm{ID} / \mathrm{cm}^{3}\right)$ in sham and bleomycin-treated mice for CBP7 and CBP11 from data 100 to 120 min after injection. (C) Ex vivo lung uptake in sham and bleomycin-treated mice at 150 min after CBP7 and CBP11 injection. $\mathrm{BM}=$ bleomycin.

contains 3 gadolinium chelates to overcome the sensitivity limitations of MRI. However, for a PET probe, only 1 imaging reporter is required. We reviewed the structure-activity data reported in the patent covering EP-3533 and chose the peptides used in CBP1, CBP3, CBP5, CBP6, and CBP7. Each peptide contains 17 amino acids, of which 11 are conserved to maintain collagen affinity. The disulfide bond was also shown to be essential (4). Positions X5, X7, X14, X15, and X16 were varied. These peptides, derivatized with gadolinium-diethylenetriamine pentaacetic acid at the $\mathrm{N}$ terminus, were shown to have an affinity similar to type I collagen (18). We replaced the thiourea-conjugated diethylenetriamine pentaacetic acid with the NODAGA chelator linked via an amide bond. CBP1 and CBP6 had the same overall charge but different lipophilic groups at different positions. CBP3 possessed an additional negative charge due to the presence of aspartic acid. For CBP6, an arginine was used to introduce an additional positive charge. We reasoned that varying the polarity, charge, and lipophilicity of the peptide would lead to differences in metabolic stability and off-target distribution when assessed in vivo. As a reference, we synthesized CBP7, which has the same amino acid sequence as EP-3533 but with 3 NODAGA chelators instead of gadoliniumdiethylenetriamine pentaacetic acid moieties.
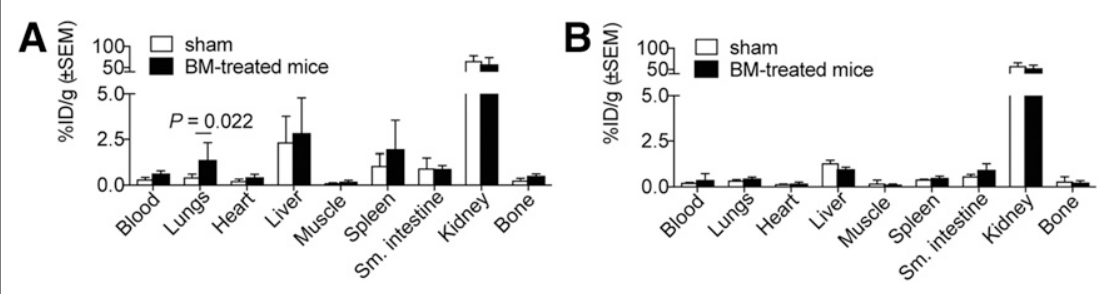

FIGURE 5. Biodistribution for CBP7 ( $n=7 /$ group) (A) and CBP11 ( $n=4 /$ group) (B) in sham and bleomycin-treated mice, $120 \mathrm{~min}$ after probe injection. $\mathrm{BM}=$ bleomycin.
We chose ${ }^{64} \mathrm{Cu}$ to take advantage of the favorable nuclear characteristics of this radioisotope and its availability with high specific activity (20). In a practical sense, ${ }^{64} \mathrm{Cu}$ is a convenient radioisotope for preclinical screening. The 12.7 -h half-life of ${ }^{64} \mathrm{Cu}$ provides the flexibility to prepare doses for whole cohorts of mice in a single labeling. The long half-life of ${ }^{64} \mathrm{Cu}$ is also suitable for peptide-based probes, which normally require a flexible radiolabeling condition and longer circulation times to achieve optimal targeting and uptake (21). The NOTA family of chelators can be easily labeled in mild conditions, and the resulting complexes are resistant to transmetallation (12).

As expected from the previous structureactivity relationship data, all the probes exhibited a low micromolar affinity for collagen. Although CBP1, CBP3, and CBP7 showed an affinity comparable to that of the established probe EP-3533, the collagen affinity of CBP5 and CBP6 was somewhat lower. Although all 5 probes displayed collagen affinity in the low micromolar range, their performance in the bleomycin mouse model of pulmonary fibrosis was diverse. The pharmacokinetic profile was similar for all the probes in terms of blood clearance of radioactivity, but the metabolic stability varied substantively from one probe to another, with CBP7 being the most metabolically stable.

An unexpected finding in this work was that the peptide with 3 NODAGA chelators (CBP7) showed much higher uptake in fibrotic lung than the peptides with 1 NODAGA chelator. This is likely due to the greater in vivo metabolic stability associated with this probe. The 3 large hydrophilic chelators in CBP7 appear to block peptidase activity. CBP7 also contains an unnatural amino acid (4,4'-biphenylalanine) at the $\mathrm{C}$ terminus, but we note that $\mathrm{CBP} 3$ also contained an unnatural amino acid (2-napthylalanine) at its $\mathrm{C}$ terminus but CBP3 was much less stable in vivo. In a different family of peptides, we previously showed that conjugating a chelating group at both the $\mathrm{C}$ and the $\mathrm{N}$ terminus of the peptide effectively blocked peptidase degradation (22). However, CBP7 showed remarkably high metabolic stability despite the absence of a chelator at the C terminus. CBP7 remained intact in the blood over time, which allows for greater accumulation in fibrotic tissues. CBP6 also showed good metabolic stability, but this was offset by 6-fold-lower collagen affinity than CBP7. The high metabolic stability of CBP7 may also explain its higher kidney retention; the intact probe is likely retained by the kidneys, whereas metabolites in the form of the simple ${ }^{64} \mathrm{Cu}$-NODAGA chelate are more likely cleared.

The goal of this work was to develop a PET imaging probe to enable early detection and staging of lung fibrosis. A successful probe must fulfill the following criteria: high uptake in the fibrotic lungs, specificity for disease, low retention in off-target organs, and high targetto-background ratios. Although it is difficult to optimize for all these parameters concurrently, the compounds synthesized in this small series could be ranked with respect to their desirability 
for further translation. CBP7 proved superior in this regard, as well as with respect to metabolic stability. CBP7 also correlates well $\left(r^{2}=\right.$ $0.83, P<0.0001$ ) with lung hydroxyproline (total collagen), demonstrating the ability of the probe to stage disease.

The specificity of CBP7 was attested by comparison with a nonbinding isomer (CBP11), wherein 1 of the cysteine moieties is changed from L-Cys in CBP7 to D-Cys in CBP11. This change in chirality resulted in a greater than 100 -fold loss in collagen affinity for CBP11; however, the distribution in normal animals was similar for both probes. The probes only behaved differently in bleomycintreated mice, with CBP7 highly accumulated in fibrotic lungs.

There has been increasing interest in molecular imaging of pulmonary fibrosis. Established probes such as the somatostatin receptor-targeted ${ }^{68} \mathrm{Ga}$-DOTANOC (23) and ${ }^{18} \mathrm{~F}$-FDG (24) have been evaluated in idiopathic pulmonary fibrosis patients. However, the specificity of these probes for idiopathic pulmonary fibrosis compared with other pulmonary pathologies, such as nonspecific interstitial pneumonia, is not clear. A cysteine-cathepsin-targeted dual optical/ PET probe ${ }^{68} \mathrm{Ga}-\mathrm{BMV} 101$ was used to monitor the contribution of inflammatory macrophages to fibrotic disease progression in a mouse model and in a small human cohort (25). Overexpression of $\alpha_{v} \beta_{6}$ integrin by alveolar epithelial cells plays a crucial role in the pathogenesis of pulmonary fibrosis (26), and an ${ }^{111} \mathrm{In}$-labeled peptide specific to $\alpha_{v} \beta_{6}$ (A20FMDV2) was used to measure $\alpha_{v} \beta_{6}$ expression in bleomycin-treated mouse lungs by SPECT (27). An ${ }^{18}$ F-labeled version of the peptide A20FMDV2 (GSK) is now under investigation in a phase 1 clinical trial (Clinicaltrials.gov identifier: NCT02052297).

The direct imaging of collagen method reported here is complementary to these techniques. Importantly, the strong correlation of lung uptake of CBP7 with hydroxyproline content indicates the ability of this probe to stage pulmonary fibrosis.

One limitation of this work was the high kidney retention of CBP7 (\%ID/g > 60) in mice, although the uptake in rat kidneys was much lower $(9.2 \% \mathrm{ID} / \mathrm{g})$. The 12.7 -h half-life of ${ }^{64} \mathrm{Cu}$ would result in a greater radiation exposure to the kidney. In this regard, using a radionuclide with a shorter half-life such ${ }^{68} \mathrm{Ga}(68 \mathrm{~min})$ or ${ }^{18} \mathrm{~F}$ (110 $\left.\mathrm{min}\right)$ may be preferred for translation to humans $(28)$. Work in that direction is ongoing.

\section{CONCLUSION}

We synthesized and evaluated 5 new collagen-targeted PET probes in a murine model of pulmonary fibrosis. Our best probe, CBP7, showed a fast blood clearance, a high stability with respect to metabolism, and a high target-to-background ratio and is specific for collagen. CBP7 represents a promising candidate for clinical translation of pulmonary fibrosis imaging.

\section{DISCLOSURE}

This project was supported by the National Heart and Lung and Blood Institute (NHLBI) (R01HL116315, R01HL131907). Pauline Désogère was supported by the Harvard/MGH Nuclear Medicine Training program, funded by the U.S. Department of Energy (DE-SC0008430). Instrumentation support from the National Center for Research Resources (S10RR029495) and the Office of Director (S10OD010650) is acknowledged. Peter Caravan has equity in Collagen Medical, the company that holds the patent rights to the peptides used in these probes. Peter Caravan and Pauline Désogère are inventors on patent/patent application (WO 2015196208) submitted by Massachusetts General Hospital that covers all probes. No other potential conflict of interest relevant to this article was reported.

\section{REFERENCES}

1. Vancheri C, Failla M, Crimi N, et al. Idiopathic pulmonary fibrosis: a disease with similarities and links to cancer biology. Eur Respir J. 2010;35:496-504.

2. Wynn TA, Ramalingam TR. Mechanisms of fibrosis: therapeutic translation for fibrotic disease. Nat Med. 2012;18:1028-1040.

3. Zhou Y, Chen H, Ambalavanan N, et al. Noninvasive imaging of experimental lung fibrosis. Am J Respir Cell Mol Biol. 2015;53:8-13.

4. Caravan P, Das B, Dumas S, et al. Collagen-targeted MRI contrast agent for molecular imaging of fibrosis. Angew Chem Int Ed Engl. 2007;46:8171-8173.

5. Helm PA, Caravan P, French BA, et al. Postinfarction myocardial scarring in mice: molecular MR imaging with use of a collagen-targeting contrast agent. Radiology. 2008;247:788-796.

6. Polasek M, Fuchs BC, Uppal R, et al. Molecular MR imaging of liver fibrosis: a feasibility study using rat and mouse models. J Hepatol. 2012;57:549-555.

7. Farrar CT, DePeralta DK, Day H, et al. 3D molecular MR imaging of liver fibrosis and response to rapamycin therapy in a bile duct ligation rat model. J Hepatol. 2015;63:689-696.

8. Zhu B, Wei L, Rotile N, et al. Combined magnetic resonance elastography and collagen molecular magnetic resonance imaging accurately stage liver fibrosis in a rat model. Hepatology. 2017;65:1015-1025.

9. Caravan P, Yang Y, Zachariah R, et al. Molecular magnetic resonance imaging of pulmonary fibrosis in mice. Am J Respir Cell Mol Biol. 2013;49:1120-1126.

10. Blodgett TM, Meltzer CC, Townsend DW. PET/CT: form and function. Radiology. 2007;242:360-385.

11. Levy SG, Jacques V, Zhou KL, et al. Development of a multigram asymmetric synthesis of 2-(R)-2-(4,7,10-tris tert-Butylcarboxymethyl-1,4,7,10-tetraazacyclododec-1yl)-pentanedioic acid, 1-tert-butyl ester, (R)-tert-bu4-DOTAGA. Org Process Res Dev. 2009;13:535-542.

12. Blasi F, Oliveira BL, Rietz TA, et al. Effect of chelate type and radioisotope on the imaging efficacy of four fibrin-specific PET probes. J Nucl Med. 2014;55:1157-1163.

13. National Research Council. Guide for the Care and Use of Laboratory Animals: Eighth Edition. Washington, D.C.: National Academies Press; 2011.

14. McGrath JC, Drummond G, McLachlan E, et al. Guidelines for reporting experiments involving animals: the ARRIVE guidelines. Br J Pharmacol. 2010;160:1573-1576.

15. Moeller A, Ask K, Warburton D, et al. The bleomycin animal model: a useful tool to investigate treatment options for idiopathic pulmonary fibrosis? Int $J$ Biochem Cell Biol. 2008;40:362-382.

16. Loening AM, Gambhir SS. AMIDE: a free software tool for multimodality medical image analysis. Mol Imaging. 2003;2:131-137.

17. Hutson PR, Crawford ME, Sorkness RL. Liquid chromatographic determination of hydroxyproline in tissue samples. J Chromatogr B Analyt Technol Biomed Life Sci. 2003;791:427-430.

18. Caravan PD, Kolodziej A, Zhang Z, et al., inventors; Collagen Medical LLC, assignee. Methods for collagen imaging. U.S. Patent 8,034,898. Published October 11, 2011.

19. Srivastava AK. Hydroxyproline: a potential biochemical marker and its role in the pathogenesis of different diseases. Curr Protein Pept Sci. 2016;17:596-602.

20. Wadas TJ, Wong EH, Weisman GR, et al. Coordinating radiometals of copper, gallium, indium, yttrium, and zirconium for PET and SPECT imaging of disease. Chem Rev. 2010;110:2858-2902.

21. Fichna J, Janecka A. Synthesis of target-specific radiolabeled peptides for diagnostic imaging. Bioconjug Chem. 2003;14:3-17.

22. Zhang Z, Kolodziej AF, Qi J, et al. Effect of peptide-chelate architecture on metabolic stability of peptide-based MRI contrast agents. New J Chem. 2010;2010:611-616.

23. Ambrosini V, Zompatori M, De Luca F, et al. ${ }^{68} \mathrm{Ga}$-DOTANOC PET/CT allows somatostatin receptor imaging in idiopathic pulmonary fibrosis: preliminary results. J Nucl Med. 2010;51:1950-1955.

24. Groves AM, Win T, Screaton NJ, et al. Idiopathic pulmonary fibrosis and diffuse parenchymal lung disease: implications from initial experience with ${ }^{18} \mathrm{~F}-\mathrm{FDG}$ PET/CT. J Nucl Med. 2009;50:538-545.

25. Withana NP, Ma X, McGuire HM, et al. Non-invasive imaging of idiopathic pulmonary fibrosis using cathepsin protease probes. Sci Rep. 2016;6:19755.

26. Munger JS, Huang X, Kawakatsu H, et al. A mechanism for regulating pulmonary inflammation and fibrosis: the integrin $\alpha_{\mathrm{v}} \beta_{6}$ binds and activates latent TGFß. Cell. 1999;96:319-328.

27. John AE, Luckett JC, Tatler AL, et al. Preclinical SPECT/CT imaging of $\alpha v \beta 6$ integrins for molecular stratification of idiopathic pulmonary fibrosis. $\mathrm{J} \mathrm{Nucl}$ Med. 2013;54:2146-2152.

28. Désogère P, Tapias LF, Hariri LP, et al. Type I collagen-targeted PET probe for pulmonary fibrosis detection and staging in preclinical models. Sci Transl Med. 2017;9:eaaf4696. 